\title{
Domain Decomposition Modified with Characteristic Mixed Finite Element and Numerical Analysis for Three-Dimensional Slightly Compressible Oil-Water Seepage Displacement
}

\author{
Yirang Yuan ${ }^{1}$, Luo Chang ${ }^{1}$, Changfeng Li $^{1,2} \&$ Tongjun Sun ${ }^{1}$ \\ ${ }^{1}$ Institute of Mathematics, Shandong University, Jinan, China \\ ${ }^{2}$ School of Economics, Shandong University, Jinan, China \\ Correspondence: Yirang Yuan, Institute of Mathematics, Shandong University, Jinan, ShandaNanlu 27, China. Tel: 86- \\ 531-88364732. E-mail: yryuan@sdu.edu.cn
}

Received: November 26, 2016 Accepted: December 28, 2016 Online Published: January 23, 2017

doi:10.5539/jmr.v9n1p143 URL: http://dx.doi.org/10.5539/jmr.v9n1p143

The research is financed by the National Natural Science Foundation of China (Grant Nos: 11101244, 11271231), Natural Science Foundation of Shandong Province (Grant No. ZR2016AM08)

\begin{abstract}
A parallel algorithm is presented to solve three-dimensional slightly compressible seepage displacement where domain decomposition and characteristics-mixed finite element are combined. Decomposing the computational domain into several subdomains, we define a special function to approximate the derivative at interior boundary explicitly and obtain numerical solutions of the saturation implicitly on subdomains in parallel. The method of characteristics can confirm strong stability at the fronts, and can avoid numerical dispersion and nonphysical oscillation. It can adopt large-time step but can obtain small time truncation error. So a characteristic domain decomposition finite element scheme is put forward to compute the saturation. The flow equation is computed by the method of mixed finite element and numerical accuracy of Darcy velocity is improved one order. For a model problem we apply some techniques such as variation form, domain decomposition, the method of characteristics, the principle of energy, negative norm estimates, induction hypothesis, and the theory of priori estimates of differential equations to derive optimal error estimate in $l^{2}$ norm. Numerical example is given to testify theoretical analysis and numerical data show that this method is effective in solving actual applications. Then it can solve the well-known problem.
\end{abstract}

Keywords: slightly compressible oil-water seepage displacement, domain decomposition parallel computation, characteristics-mixed finite element, optimal error estimate in $l^{2}$ norm, numerical computation and analysis

\section{Mathematical Model and Physical Background}

High-pressure pump injects water into oil storage and displaces crude oil out from production wells. This technique is popular and important in modern oil exploration. The displacement of two phase describes the physical phenomena how injected water displaces crude oil in reservoir and crude oil is produced. In modern oil recovery we try to make remaining crude oil out by adopting a third-recovery technique (chemical displacement). It is necessary to consider the compressibility in numerical simulation to avoid numerical distortion. Douglas and other scholars put forward a miscible mathematical model with slight-compressibility and discuss the methods of characteristic finite element and characteristic mixed element, then they give the outline of modern numerical simulation in oil recovery (Douglas, \& Roberts, 1983; Ewing, 1983; Yuan, 1992,1993,2013).

The mathematical model is stated by the following nonlinear system of partial differential equations with initial-boundary value conditions (Douglas, \& Roberts, 1983; Ewing, 1983; Yuan, 1992,1993,2013):

$$
\begin{aligned}
& d(c) \frac{\partial p}{\partial t}+\nabla \cdot \mathbf{u}=d(c) \frac{\partial p}{\partial t}-\nabla \cdot(a(c) \nabla p)=q(X, t), X=(x, y, z)^{T} \in \Omega, t \in J=(0, T], \\
& \mathbf{u}=-a(c) \nabla p, X \in \Omega, t \in J \\
& \phi \frac{\partial c}{\partial t}+b(c) \frac{\partial p}{\partial t}+\mathbf{u} \cdot \nabla c-\nabla \cdot(D \nabla c)=(\tilde{c}-c) \tilde{q}, X \in \Omega, t \in J,
\end{aligned}
$$

where $\Omega$ is a bounded domain in $R^{3} . d(c)=\phi(X) \sum_{j=1}^{2} z_{j} c_{j}, b(c)=\phi(X) c_{i}\left\{z_{1}-\sum_{j=1}^{2} z_{j} c_{j}\right\}$, where $c_{1}, c_{2}$ denote two different components of the saturation, and $z_{1}, z_{2}$ denote the compressibility. Let $c=c_{1}=1-c_{2}$ denote the first component of the 
saturation at production well. The given function $\bar{c}$ denotes the saturation of injection well. $k(X)$ denotes the permeability and $\mu(c)$ is the viscosity, then $a(c)=k(X) \mu^{-1}(c)$. The porosity, the pressure and the production rate are denoted by $\phi=\phi(X), p(X, t)$ and $q(X, t)$, respectively. $\tilde{q}=\max \{q, 0\}$. $\mathbf{u}=\mathbf{u}(X, t)$ is Darcy velocity and $D=\phi(X) d_{m} I$, where $d_{m}$ is diffusion coefficient and $I$ is a unit matrix.

Suppose that the fluid is not permeable at the boundary, that is to say that the following conditions hold

$$
\mathbf{u} \cdot v=0, X \in \partial \Omega, t \in J,(D \nabla c-c \mathbf{u}) \cdot v=0, X \in \partial \Omega, t \in J,
$$

where $v$ is the normal vector of $\partial \Omega$, the boundary surface of $\Omega$.

Initial conditions are defined by

$$
p(X, 0)=p_{0}(X), X \in \Omega, \quad c(X, 0)=c_{0}(X), X \in \Omega .
$$

For two-dimensional incompressible two-phase seepage displacement, Douglas and Ewing et. al. put forward characteristic finite difference and characteristic finite element for periodic problems and give rigorous convergence analysis (Douglas, \& Russell, 1982; Douglas, \& Yuan, 1986; Ewing, \& Russell, 1983; Ewing, Yuan, \& Li,1989; Russell, 1985). Combining normal finite difference or finite element with the method of characteristics, they present two different composite schemes. These schemes can reflect the first-order hyperbolic nature of convection-diffusion equation, decrease truncation error, overcome numerical oscillation and dispersion and they can improve the stability and accuracy greatly. The compressibility must be considered in new numerical simulation of modern enhanced oil displacement (Douglas, \& Roberts, 1983; Yuan, 1992,2013). Under periodic assumption Douglas and Yuan firstly discuss characteristic finite element and characteristic mixed finite element, obtain optimal order error estimate in $L^{2}$ norm and give a powerful tool to solve the well-known problem (Douglas, \& Roberts, 1983; Yuan, 1999,2003).

In numerical simulation of modern oil field exploration and development (special for enhanced chemical recovery), the computation is large-scaled, and it runs not only on a three-dimensional domain but also on a long time interval. Its nodes amount up to tens of thousands or millions, therefore traditional methods can not solve this problem well. So new modern parallel computation methods should be introduced (Ewing, 1983; Shen, Liu, \& Tang, 2002). Dawson, Dupont and Du firstly present Galerkin domain decomposition procedure and give convergence analysis (Dawson, \& Du, 1990; Dawson, Du, \& Dupont, 1991; Daswon, \& Dupont, 1992,1994) for a simple parabolic equation. For heat conductor transient problem we have published many research results (Yuan, Chang, Li, \& Sun, 2015) about domain decomposition modified by characteristic finite element and characteristic mixed finite element. We have considered the enhanced oil recovery simulation with incompressible condition and give the primary study (Yuan, Chang, Li, \& Sun, 2015). Since computational task of the saturation is dominated and large-scaled in the whole numerical simulation, so parallel computation of the saturation is considered mainly in this paper. Based on the above research we put forward a modified characteristic domain decomposition method to solve three-dimensional compressible seepage displacement in this paper. Decomposing computational domain into several subdomains, we define a special function to approximate the value at interior boundary explicitly and obtain numerical solution implicitly in parallel in subdomains. The flow equation is discretized by the method of mixed finite element and the saturation is approximated by a domain decomposition scheme of modified characteristic finite element. For the model problem we use variation form, domain decomposition, the characteristics, the principle of energy, induction hypothesis, the theory and technique of priori estimates of partial differential equations to get optimal order error estimate in $L^{2}$ norm. Numerical experiment is consistent with theoretical analysis and confirms that the method is efficient and feasible in actual computation. It is an important and powerful tool in model analysis, numerical method, principle research and engineering applicable software design of modern oil reservoir exploration and development and it can solve the well-known problem (Douglas, \& Roberts, 1983; Ewing, 1983; Shen, Liu, \& Tang, 2002).

Suppose that exact solutions of (1)-(4) are sufficiently smooth and the coefficients are positive definite

$$
0<\phi_{*} \leq \phi(X) \leq \phi^{*}, 0<a_{*} \leq a(c) \leq a^{*}, 0<d_{*} \leq d(c) \leq d^{*}, 0<D_{*} \leq D(X) \leq D^{*},
$$

where $\phi_{*}, \phi^{*}, a_{*}, a^{*}, d_{*}, d^{*}, D_{*}$ and $D^{*}$ are positive constants.

For simplicity we suppose that (1)-(4) is $\Omega$-periodic (Douglas, \& Yuan, 1986; Ewing, \& Russell, 1982; Ewing, Yuan, $\& \mathrm{Li}, 1989$; Russell, 1985), that is, all the functions are supposed to be $\Omega$-periodic. This assumption is reasonable in physical science and boundary condition is usually used. In numerical simulation of oil reservoir, boundary condition affects the interior flow slightly, therefore the condition (3) can be dropped (Douglas, \& Yuan, 1986; Ewing, \& Russell, 1982; Ewing, Yuan, \& Li, 1989; Russell, 1985). 
In the following discussion the symbols $K$ and $\varepsilon$ denote a generic positive constant and a generic small positive number, respectively. They have different definitions at different places.

\section{Some Preliminary Notations}

For simplicity, decompose $\Omega=\left\{\left(x_{1}, x_{2}, x_{3}\right) \mid 0<x_{1}<1,0<x_{2}<1,0<x_{3}<1\right\}$ into two subdomains $\Omega_{1}=\left\{\left(x_{1}, x_{2}, x_{3}\right) \mid 0<\right.$ $\left.x_{1}<1 / 2,0<x_{2}<1,0<x_{3}<1\right\}, \Omega_{2}=\left\{\left(x_{1}, x_{2}, x_{3}\right) \mid 1 / 2<x_{1}<1,0<x_{2}<1,0<x_{3}<1\right\}, \Gamma=\left\{\left(x_{1}, x_{2}, x_{3}\right) \mid x_{1}=1 / 2,0<\right.$ $\left.x_{2}<1,0<x_{3}<1\right\}$ (see Figure. 1).

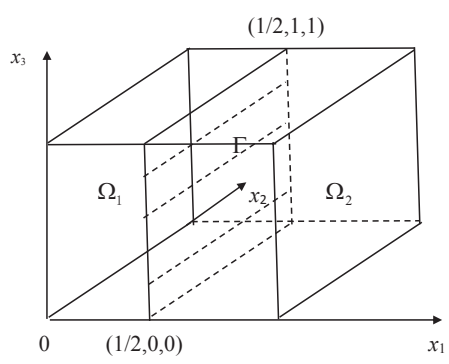

Figure 1. Partition of domain decomposition $\Omega_{1}, \Omega_{2}, \Gamma$

To approximate the normal derivative at $\Gamma$, we define two special functions $\Phi_{2}$ and $\Phi_{4}$ as follows (Dawson, \& Du, 1990; Dawson, \& Dupont, 1992),

$$
\begin{aligned}
\Phi_{2}\left(x_{1}\right) & = \begin{cases}1-x_{1}, & 0 \leq x_{1} \leq 1 \\
x_{1}+1, & -1 \leq x_{1} \leq 0, \\
0, & \text { otherwise }\end{cases} \\
\Phi_{4}\left(x_{1}\right) & = \begin{cases}\left(x_{1}-2\right) / 12, & 1 \leq x_{1} \leq 2, \\
-5 x_{1} / 4+7 / 6, & 0 \leq x_{1} \leq 1, \\
5 x_{1} / 4+7 / 6, & -1 \leq x_{1} \leq 0, \\
-\left(x_{1}+2\right) / 12, & -2 \leq x_{1} \leq-1, \\
0, & \text { otherwise }\end{cases}
\end{aligned}
$$

Note that if $p\left(x_{1}\right)$ is a polynomial of degree at most one, then

$$
\int_{-\infty}^{\infty} p\left(x_{1}\right) \Phi_{2}\left(x_{1}\right) d x_{1}=p(0)
$$

and if $p\left(x_{1}\right)$ is a polynomial of degree at most three, then

$$
\int_{-\infty}^{\infty} p\left(x_{1}\right) \Phi_{4}\left(x_{1}\right) d x_{1}=p(0) .
$$

Definition 1: For $H \in\left(0, \frac{1}{2}\right)$, define

$$
\Phi\left(x_{1}\right)=\Phi_{m}\left(\left(x_{1}-1 / 2\right) / H\right) / H, m=2,4 .
$$

Let $N_{h, j}$ denote a finite-dimensional finite element space of $H^{1}\left(\Omega_{j}\right)(j=1,2)$, and let $N_{h}(\Omega)$ be an $l$-dimensional subspace of $L^{2}(\Omega)$. Moreover, for a function $v \in N_{h}$, then $\left.v\right|_{\Omega_{j}} \in N_{h, j}$. [v], the jump of $v \in N_{h}(\Omega)$ at interior boundary $\Gamma$, is defined by

$$
[v]_{\left(\frac{1}{2}, x_{2}, x_{3}\right)}=v\left(\frac{1}{2}+0, x_{2}, x_{3}\right)-v\left(\frac{1}{2}-0, x_{2}, x_{3}\right) .
$$

Definition 2: A bilinear function $\bar{D}(u, v)$ is defined by

$$
\bar{D}(u, v)=\int_{\Omega_{1} \cup \Omega_{2}} D(X) \nabla u \cdot \nabla v d x_{1} d x_{2} d x_{3}+\lambda \int_{\Omega_{1} \cup \Omega_{2}} u v d x_{1} d x_{2} d x_{3},
$$

where $u, v \in H^{1}\left(\Omega_{j}\right), j=1,2 . D(X)$ is a positive definite function, $D_{T}=1$ and $\lambda_{s}$ is a positive constant.

Definition 3: An integral operator is defined to approximate normal derivative at interior boundary,

$$
B(\psi)\left(\frac{1}{2}, x_{2}, x_{3}\right)=-\int_{0}^{1} \Phi^{\prime}\left(x_{1}\right) \psi\left(x_{1}, x_{2}, x_{3}\right) d x_{1},
$$


where $\Phi\left(x_{1}\right)$ is defined by (7).

Let $(\cdot, \cdot)$ denote inner product in $L^{2}\left(\Omega_{1} \cup \Omega_{2}\right)$, and omit the subscript $(\psi, \rho)=(\psi, \rho)_{\Omega}$ on $\Omega=\Omega_{1} \cup \Omega_{2}$. For a function $\psi$ in $H^{1}\left(\Omega_{1}\right)$ and $H^{1}\left(\Omega_{2}\right)$, define

$$
\|\psi \psi\|^{2}=\bar{D}(\psi, \psi)+H^{-1}\|D[\psi]\|_{L^{2}(\Gamma)}^{2} .
$$

Noting that

$$
\begin{aligned}
\left(D\left(x_{1}, x_{2}, x_{3}\right) B(\psi),[\psi]\right)_{\Gamma} & =-\int_{0}^{1} \int_{0}^{1} D\left(\frac{1}{2}, x_{2}, x_{3}\right) \int_{0}^{1} \Phi^{\prime}\left(x_{1}\right) \psi\left(x_{1}, x_{2}, x_{3}\right) d x_{1}[\psi]\left(\frac{1}{2}, x_{2}, x_{3}\right) d x_{2} d x_{3} \\
\int_{0}^{1} \Phi^{\prime}\left(x_{1}\right) \psi\left(x_{1}, x_{2}, x_{3}\right) d x_{1} & =\left.\psi\left(x_{1}, x_{2}, x_{3}\right) \Phi\left(x_{1}\right)\right|_{0} ^{1}-\int_{0}^{1} \Phi\left(x_{1}\right) \psi_{x_{1}}\left(x_{1}, x_{2}, x_{3}\right) d x_{1} \\
& =-\frac{1}{H}[\psi]\left(\frac{1}{2}, x_{2}, x_{3}\right)-\int_{0}^{1} \Phi\left(x_{1}\right) \psi_{x_{1}}\left(x_{1}, x_{2}, x_{3}\right) d x_{1}
\end{aligned}
$$

then, we have

$$
\begin{aligned}
\left(D\left(x_{1}, x_{2}, x_{3}\right) B(\psi),[\psi]\right)_{\Gamma}= & \frac{1}{H} \int_{0}^{1} \int_{0}^{1} D\left(\frac{1}{2}, x_{2}, x_{3}\right)[\psi]^{2}\left(\frac{1}{2}, x_{2}, x_{3}\right) d x_{2} d x_{3} \\
& +\int_{0}^{1} \int_{0}^{1} D\left(\frac{1}{2}, x_{2}, x_{3}\right) \int_{0}^{1} \Phi\left(x_{1}\right) \psi_{x_{1}}\left(x_{1}, x_{2}, x_{3}\right) d x_{1}[\psi]\left(\frac{1}{2}, x_{2}, x_{3}\right) d x_{2} d x_{3} .
\end{aligned}
$$

Rewrite the second term of the above expression as follows,

$$
\begin{aligned}
& \int_{0}^{1} \int_{0}^{1} D^{1 / 2}\left(\frac{1}{2}, x_{2}, x_{3}\right) \int_{\frac{1}{2}-H}^{\frac{1}{2}+H} D^{1 / 2}\left(\frac{1}{2}, x_{2}, x_{3}\right) \Phi\left(x_{1}\right) \psi_{x_{1}}\left(x_{1}, x_{2}, x_{3}\right) d x_{1}[\psi]\left(\frac{1}{2}, x_{2}, x_{3}\right) d x_{2} d x_{3} \\
& \leq \int_{0}^{1} \int_{0}^{1} D^{1 / 2}\left(\frac{1}{2}, x_{2}, x_{3}\right)\left(\int_{0}^{1} \Phi^{2}\left(x_{1}\right) d x_{1}\right)^{1 / 2}\left(\int_{\frac{1}{2}-H}^{\frac{1}{2}+H} D\left(\frac{1}{2}, x_{2}, x_{3}\right) \psi_{x_{1}}^{2}\left(x_{1}, x_{2}, x_{3}\right) d x_{1}\right)^{1 / 2} \\
& \quad \cdot[\psi]\left(\frac{1}{2}, x_{2}, x_{3}\right) d x_{2} d x_{3} \\
& \leq\left(\frac{2}{3 H}\right)^{1 / 2}\left(\int_{0}^{1} \int_{0}^{1} D\left(\frac{1}{2}, x_{2}, x_{3}\right)[\psi]^{2}\left(\frac{1}{2}, x_{2}, x_{3}\right) d x_{2} d x_{3}\right)^{1 / 2} \\
& \quad \cdot\left(\int_{0}^{1} \int_{0}^{1} \int_{\frac{1}{2}-H}^{\frac{1}{2}+H} D\left(\frac{1}{2}, x_{2}, x_{3}\right) \psi_{x_{1}}^{2}\left(x_{1}, x_{2}, x_{3}\right) d x_{1} d x_{2} d x_{3}\right)^{1 / 2} .
\end{aligned}
$$

Note an equivalent formulation of $D\left(\frac{1}{2}, x_{2}, x_{3}\right)$,

$$
D\left(\frac{1}{2}, x_{2}, x_{3}\right)=D\left(x_{1}, x_{2}, x_{3}\right)+\left(x_{1}-\frac{1}{2}\right) \frac{\partial D}{\partial x_{1}}\left(\xi_{1}\left(x_{1}\right), x_{2}, x_{3}\right),
$$

where $\left(x_{1}-\frac{1}{2}\right) \frac{\partial p}{\partial x_{1}}\left(\xi_{1}(X), x_{2}, x_{3}\right)$ is the value of Taylor remainder and $\xi_{1}(X)$ is a point between $\frac{1}{2}$ and $x_{1}$. Then

$$
\begin{aligned}
& \int_{0}^{1} \int_{0}^{1} \int_{\frac{1}{2}-H}^{\frac{1}{2}+H} D\left(\frac{1}{2}, x_{2}, x_{3}\right) \psi_{x_{1}}^{2}\left(x_{1}, x_{2}, x_{3}\right) d x_{1} d x_{2} d x_{3} \\
& =\int_{0}^{1} \int_{0}^{1} \int_{\frac{1}{2}-H}^{\frac{1}{2}+H}\left[D\left(x_{1}, x_{2}, x_{3}\right)+\left(x_{1}-\frac{1}{2}\right) \frac{\partial D}{\partial x_{1}}\left(\xi_{1}\left(x_{1}\right), x_{2}, x_{3}\right)\right] \psi_{x_{1}}^{2}\left(x_{1}, x_{2}, x_{3}\right) d x_{1} d x_{2} d x_{3} \\
& \leq\left(1+M^{*} H\right) \int_{0}^{1} \int_{0}^{1} \int_{\frac{1}{2}-H}^{\frac{1}{2}+H} D\left(x_{1}, x_{2}, x_{3}\right) \psi_{x_{1}}^{2}\left(x_{1}, x_{2}, x_{3}\right) d x_{1} d x_{2} d x_{3},
\end{aligned}
$$

where $M^{*}=\max _{\substack{x_{1} \in\left(\frac{1}{2}-H, \frac{1}{2}+H\right) \\\left(x_{2}, x_{3}\right) \in(0,1) \times(0,1)}} \frac{\left|\frac{\partial D}{\partial x_{1}}\left(\xi\left(x_{1}\right), x_{2}, x_{3}\right)\right|}{D\left(x_{1}, x_{2}, x_{3}\right)}$.

Therefore, there exists a positive constant $M_{0}$ such that

$$
\bar{D}(\psi, \psi)+(D B(\psi),[\psi])_{\Gamma} \geq \frac{1}{M_{0}}\|\psi\|^{2},
$$


i.e.,

$$
\|\mid \psi\|^{2} \leq M_{0}\left\{\bar{D}(\psi, \psi)+(D B(\psi),[\psi])_{\Gamma}\right\}
$$

Similarly, we have the following estimates for $0 \leq t \leq T$

$$
\begin{aligned}
& \|B(\psi)\|_{L^{2}(\Gamma)}^{2} \leq M_{1} H^{-3}\|\psi\|_{0}^{2}, \\
& \|B(\psi)\|_{L^{2}(\Gamma)} \leq M_{2} H^{-1}\|\psi\|_{0, \infty}, \\
& \left\|\frac{\partial u(\cdot, t)}{\partial \gamma}-B(u)(\cdot, t)\right\|_{L^{2}(\Gamma)} \leq M_{3} H^{m},
\end{aligned}
$$

where $M_{1}, M_{2}, M_{3}$ are positive constants, $m=2,4$, and $\frac{\partial u}{\partial \gamma}$ denotes the normal derivative of $u$ across interior boundary $\Gamma$.

\section{Modified Characteristic Mixed Finite Element Domain Decomposition Procedure}

The variation of (2) is defined by

$$
\begin{aligned}
& \left(\phi \frac{\partial c}{\partial t}, v\right)+(\mathbf{u} \cdot \nabla c, v)+(D \nabla c, \nabla v)+\left(b(c) \frac{\partial p}{\partial t}, v\right)+\left(D \frac{\partial c}{\partial n},[v]\right)_{\Gamma}=(g(c), v), v \in N(\Omega), \\
& c(X, 0)=c_{0}(X), X \in \Omega,
\end{aligned}
$$

where $(\psi, v)=\int_{\Omega_{1} \cup \Omega_{2}} \psi v d x_{1} d x_{2} d x_{3},(\psi, v)_{\Gamma}=\int_{\Gamma} \psi v d x_{2} d x_{3}, g(c)=(\tilde{c}-c) q$.

The pressure is solved by the method of mixed finite element. For a vector $f=\left(f_{1}, f_{2}, f_{3}\right)^{T}$, define two spaces $\tilde{H}(\operatorname{div}, \Omega)=$ $\left\{f: f_{1}, f_{2}, f_{3}, \nabla \cdot f \in L^{2}(\Omega)\right.$, periodic $\}$ and $\tilde{L}^{2}(\Omega)=\left\{g: g \in L^{2}(\Omega)\right.$, periodic $\}$. For convenience we omit the symbol " " and let $V=H(\operatorname{div} ; \Omega), W=L^{2}(\Omega)$. Considering the equations on $\Omega_{1}, \Omega_{2}$, we introduce the following compatibility condition

$$
p_{1}=p_{2}, \quad \mathbf{u}_{1} \cdot n_{1}+\mathbf{u}_{2} \cdot n_{2}=0, \quad X \in \Gamma,
$$

where $n_{1}, n_{2}$ are unit normal directions of $\Omega_{1}, \Omega_{2}$ at $\Gamma$. Let $W_{i}=L^{2}\left(\Omega_{i}\right), V_{i}=H\left(\operatorname{div} ; \Omega_{i}\right)$ and $\Lambda=\left\{v:\left.v\right|_{\Gamma} \in L^{2}(\Gamma), \Gamma \neq \varnothing\right\}$, then we get the following variation of (1) on subdomains $\Omega_{i}(i=1,2)$

$$
\begin{aligned}
& \left(d(c) \frac{\partial p}{\partial t}, w\right)_{\Omega_{i}}+(\nabla \cdot \mathbf{u}, w)_{\Omega_{i}}=(q, w)_{\Omega_{i}}, \forall w \in W_{i}, \\
& \left(a^{-1}(c) \mathbf{u}, z\right)_{\Omega_{i}}-(\nabla \cdot z, p)_{\Omega_{i}}+\left(p, z \cdot n_{i}\right)_{\Gamma}=0, \forall z \in V_{i}, \\
& \left(\beta, \mathbf{u}_{1} \cdot n_{1}+\mathbf{u}_{2} \cdot n_{2}\right)_{\Gamma}=0, \forall \beta \in \Lambda,
\end{aligned}
$$

Making summation of (17a) and (17b) on $i=1,2$, we get the variation of (1) on the whole domain $\Omega$

$$
\begin{aligned}
& \left(d(c) \frac{\partial p}{\partial t}, w\right)+(\nabla \cdot \mathbf{u}, w)=(q, w), \forall w \in W, \\
& \left(a^{-1}(c) \mathbf{u}, z\right)-(\nabla \cdot z, p)+\sum_{i=1}^{2}\left(p, z^{(i)} \cdot n_{i}\right)_{\Gamma}=0, \forall z \in V, \\
& \left(\beta, \mathbf{u}_{1} \cdot n_{1}+\mathbf{u}_{2} \cdot n_{2}\right)_{\Gamma}=0, \forall \beta \in \Lambda,
\end{aligned}
$$

where $z^{(i)}=\left.z\right|_{\Gamma_{i}}$ and $\Gamma_{i}=\Gamma \cap \partial \Omega_{i}$.

The elliptic projections of the saturation, Darcy velocity and the pressure are defined as follows.

Definition 4: The elliptic projection of $c(X, t)$ is defined by $\tilde{c}(X, t): J \rightarrow N_{h}$

$$
\left(D(X)(\tilde{c}-c), \nabla v_{h}\right)+\lambda\left(\tilde{c}-c, v_{h}\right)=0, \quad \forall v_{h} \in N_{h},
$$

where $\lambda$ is a positive constant.

Definition 5: The projections of $\mathbf{u}(X, t)$ and $p(X, t)$ are defined by $\{\tilde{\mathbf{u}}, \tilde{p}\}: J \rightarrow W_{h} \times V_{h}$

$$
\begin{aligned}
& \left(d(c) \frac{\partial p}{\partial t}, w_{h}\right)+\left(\nabla \cdot \tilde{\mathbf{u}}, w_{h}\right)=\left(q, w_{h}\right), \forall w_{h} \in W_{h}, \\
& \left(a^{-1}(c) \tilde{\mathbf{u}}, v_{h}\right)-\left(\nabla \cdot v_{h}, \tilde{p}\right)=0, \forall v_{h} \in V_{h}, \\
& (\tilde{p}, 1)=(p, 1)
\end{aligned}
$$


where $W_{h} \times V_{h}$ is Raviart-Thomas space with the index $k$ and the mesh step $h_{p}$. The approximations are stated as follows (Ewing, Russell, \& Wheeler, 1984; Raviart, \& Thomas, 1977)

$$
\begin{aligned}
& \inf _{v_{h} \in V_{h}}\left\|v-v_{h}\right\|_{L^{2}(\Omega)} \leq K\|v\|_{k+2} h_{p}^{k+1}, \\
& \inf _{v_{h} \in V_{h}}\left\|\nabla \cdot\left(v-v_{h}\right)\right\|_{0} \leq K\left\{\|v\|_{k+1}+\|\nabla \cdot v\|_{k+1}\right\} h_{p}^{k+1}, \\
& \inf _{w_{h} \in W_{h}}\left\|w-w_{h}\right\|_{0} \leq K\|w\|_{k+1} h_{p}^{k+1} .
\end{aligned}
$$

Lemma 1. By using Galerkin method we get error estimate of elliptic projection for the saturation (Ewing, \& Wheeler, 1981; Wheeler, 1973) in the finite element space $N_{h}$ with the index $l$ and the mesh step $h_{c}$,

$$
\begin{aligned}
& \|c-\tilde{c}\|_{0}+h_{c}\|c-\tilde{c}\|_{1} \leq K\|c\|_{l+1} h_{c}^{l+1}, \\
& \left\|\frac{\partial(c-\tilde{c})}{\partial t}\right\|_{0}+h_{c}\left\|\frac{\partial(c-\tilde{c})}{\partial t}\right\|_{1} \leq K\left\{\|c\|_{l+1}+\left\|\frac{\partial c}{\partial t}\right\|_{l+1}\right\} h_{c}^{l+1} .
\end{aligned}
$$

Lemma 2. By Brezzi theory (Brezzi, 1974; Ciarlet, 1978) we get error estimates of mixed finite element elliptic projections for Darcy and the pressure

$$
\begin{aligned}
& \|\mathbf{u}-\tilde{\mathbf{u}}\|_{V}+\|p-\tilde{p}\|_{W} \leq K\|p\|_{k+3} h_{p}^{k+1} \\
& \left\|\frac{\partial(\mathbf{u}-\tilde{\mathbf{u}})}{\partial t}\right\|_{V}+\left\|\frac{\partial(p-\tilde{p})}{\partial t}\right\|_{W} \leq K\left\{\|p\|_{k+3}+\left\|\frac{\partial p}{\partial t}\right\|_{k+3}\right\} h_{p}^{k+1} .
\end{aligned}
$$

Considering that the fluid flows along the characteristics $\phi \rho \frac{\partial c}{\partial t}+\mathbf{u} \cdot \nabla c$, so we introduce the method of characteristics. Define $\psi=\left[\phi^{2}+|\mathbf{u}|^{2}\right]^{1 / 2}$ and $\frac{\partial}{\partial \tau}=\psi^{-1}\left\{\phi \frac{\partial}{\partial t}+\mathbf{u} \cdot \nabla\right\}$. The characteristics depends on $c, p$ and Darcy velocity $\mathbf{u}$. Then (2) is expressed by

$$
\psi \frac{\partial c}{\partial \tau}+b \frac{\partial p}{\partial t}-\nabla \cdot(D \nabla c)=g(c),(X, t) \in \Omega \times J .
$$

Let $N_{h} \subset N$ denote an $l$-dimensional subspace with mesh step $h_{c}, W_{h} \times V_{h}$ denote a $k$-order Raviart-Thomas mixed finite element space with $h_{p}$, and let $\Lambda_{h}=\left\{\beta:\left.\beta\right|_{\Gamma} \in P_{k}(\Gamma)\right\}$ denote a subspace of $\Lambda$.

The parallel procedures of characteristic mixed element are stated as follows. Given numerical solutions $\left\{P_{h}^{n}, \mathbf{U}_{h}^{n}, C_{h}^{n}\right\} \in$ $W_{h} \times V_{h} \times N_{h}$ at $t^{n}$, we find numerical solutions at $t^{n+1},\left\{P_{h}^{n+1}, \mathbf{U}_{h}^{n+1}\right\} \in W_{h} \times V_{h}, C_{h}^{n+1} \in N_{h}, n=0,1,2, \cdots$, by

$$
\begin{aligned}
& \mathbf{U}_{h}^{0}=\tilde{\mathbf{u}}^{0}, P_{h}^{0}=\tilde{p}^{0}, \\
& \left(d\left(C_{h}^{n} \frac{P_{h}^{n+1}-P_{h}^{n}}{\Delta t}, w_{h}\right)+\left(\nabla \cdot \mathbf{U}_{h}^{n+1}, w_{h}\right)=\left(q, w_{h}\right), w_{h} \in W_{h}, n \geq 0,\right. \\
& \left(a^{-1}\left(C_{h}^{n}\right) \mathbf{U}_{h}^{n}, z_{h}\right)-\left(\nabla \cdot z_{h}, P_{h}^{n+1}\right)+\sum_{i=1}^{2}\left(P_{h}^{n+1}, z_{h}^{(i)} \cdot n_{i}\right)_{\Gamma}=0, \forall z_{h} \in V_{h}, \\
& \left(\beta, \mathbf{U}_{1}^{n+1} \cdot n_{1}+\mathbf{U}_{2}^{n+1} \cdot n_{2}\right)_{\Gamma}=0, \forall \beta \in \Lambda_{h},
\end{aligned}
$$

where $\mathbf{U}_{i}^{n+1}=\mathbf{U}^{n+1}\left|\Gamma_{i}, z_{h}^{(i)}=z_{h}\right|_{\Gamma_{i}}, \Gamma_{i}=\Gamma \cap \partial \Omega_{i}, i=1,2$.

$$
\begin{aligned}
& C_{h}^{0}=\tilde{c}^{0}, \\
& \left(\phi \frac{C_{h}^{n+1}-\hat{C}_{h}^{n}}{\Delta t}, v_{h}\right)+\left(D \nabla C_{h}^{n+1}, \nabla v_{h}\right)+\left(b\left(C_{h}^{n}\right) \frac{P_{h}^{n+1}-P_{h}^{n}}{\Delta t}, v_{h}\right)+\left(D B\left(C_{h}^{n}\right),\left[v_{h}\right]\right)_{\Gamma} \\
& =\left(g\left(C_{h}^{n}\right), v_{h}\right), v_{h} \in N_{h},
\end{aligned}
$$

where $\hat{C}_{h}^{n}=C_{h}^{n}\left(\hat{X}^{n}\right), \hat{X}^{n}=X-\phi^{-1} \mathbf{U}_{h}^{n+1} \Delta t$.

The program runs as follows to obtain numerical solutions $\mathbf{U}_{h}^{0}, P_{h}^{0}, C_{h}^{0}, \mathbf{U}_{h}^{1}, P_{h}^{1}, C_{h}^{1}, \cdots$. If $\left\{\mathbf{U}_{h}^{n}, P_{h}^{n}, C_{h}^{n}\right\}$ is given at $t=t^{n}$, then we get $\left\{\mathbf{U}_{h}^{n+1}, P_{h}^{n+1}\right\}$ by (25b)-(25d). Then we get $\hat{X}^{n}$ and $\hat{C}_{h}^{n}$. It continues to compute $B\left(C_{h}^{n}\right)$ by (10). By the definition of $N_{h}=N_{h, 1} \cup N_{h, 2}$, the problem is decomposed into two independent subproblems on subdomains, then the saturation is solved in parallel. The solutions exist and are sole according to (C). 


\section{Numerical Analysis of a Model Problem}

In this section, we show how to finish the convergence analysis and error estimate of the composite method. For convenience, we only discuss a model problem by simplifying the coefficients and descriptions. Suppose that the problem is incompressible, $a(c)=k(X) \mu^{-1}(c) \approx k(X) \mu_{0}^{-1}=a(X)$, i.e. the viscosity $\mu(c)$ is approximately taken as a constant such as the displacement of lower seepage (Douglas, \& Roberts, 1983; Yuan, 2001). Then (1) and (2) are degraded into

$$
\begin{gathered}
d(c) \frac{\partial p}{\partial t}+\nabla \cdot \mathbf{u}=q, \quad(X, t) \in \Omega \times J, \\
\mathbf{u}=-a(X) \nabla p, \quad(X, t) \in \Omega \times J, \\
\phi(X) \frac{\partial c}{\partial t}+b(c) \frac{\partial p}{\partial t}+\mathbf{u} \cdot \nabla c-\nabla \cdot(D \nabla c)=g(c), \quad(X, t) \in \Omega \times J .
\end{gathered}
$$

The variation of (27) at saddle point is formulated as follows

$$
\begin{aligned}
& \left(d(c) \frac{\partial p}{\partial t}, w\right)+(\nabla \cdot \mathbf{u}, w)=(q, w), \quad \forall w \in W, \\
& \left(a^{-1} \mathbf{u}, z\right)-(\nabla \cdot z, p)=0, \quad \forall z \in V .
\end{aligned}
$$

The parallel algorithm of characteristic mixed element is defined as follows. If $\left\{P_{h}^{n}, \mathbf{U}_{h}^{n}, C_{h}^{n}\right\} \in W_{h} \times V_{h} \times N_{h}$ is given at $t=t^{n}$, then we find $\left\{P_{h}^{n+1}, \mathbf{U}_{h}^{n+1}, C_{h}^{n+1}\right\} \in W_{h} \times V_{h} \times N_{h}$ at $t=t^{n+1}$ by the following procedures

$$
\begin{aligned}
& \left(d\left(C_{h}^{n}\right) \frac{P_{h}^{n+1}-P_{h}^{n}}{\Delta t}, w_{h}\right)+\left(\nabla \cdot \mathbf{U}_{h}^{n+1}, w_{h}\right)=\left(q^{n+1}, w_{h}\right), \quad \forall w_{h} \in W_{h}, \\
& \left(a^{-1} \mathbf{U}_{h}^{n+1}, z_{h}\right)-\left(\nabla \cdot z_{h}, P_{h}^{n+1}\right)=0, \quad \forall z_{h} \in V_{h}, \\
& \left(\phi \frac{C_{h}^{n+1}-\hat{C}_{h}^{n}}{\Delta t}, v_{h}\right)+\left(D \nabla C_{h}^{n+1}, \nabla v_{h}\right)+\left(b\left(C_{h}^{n}\right) \frac{P_{h}^{n+1}-P_{h}^{n}}{\Delta t}, v_{h}\right)+\left(D B\left(C_{h}^{n}\right),\left[v_{h}\right]\right) \\
& =\left(g\left(C_{h}^{n}\right), v_{h}\right), \quad \forall v_{h} \in N_{h} .
\end{aligned}
$$

An elliptic projection of the flow is defined to find $\{\tilde{\mathbf{u}}, \tilde{p}\}: J \rightarrow W_{h} \times V_{h}$ for $t \in J=(0, T]$ satisfying the following equations

$$
\begin{aligned}
& \left(d(c) \frac{\partial p}{\partial t}, w_{h}\right)+\left(\nabla \cdot \tilde{\mathbf{u}}, w_{h}\right)=\left(q^{n+1}, w_{h}\right), \forall w_{h} \in W_{h}, \\
& \left(a^{-1} \tilde{\mathbf{u}}, z_{h}\right)-\left(\nabla \cdot z_{h}, \tilde{p}\right)=0, \forall z_{h} \in V_{h}, \\
& (\tilde{p}, 1)=(p, 1)
\end{aligned}
$$

In this section we pay more attention on convergence discussion of the scheme (30), (31) of the model (27), (28). For convenience to discuss error estimate, we denote error functions by $\zeta=c-\tilde{C}, \xi=\tilde{C}-C_{h}, \eta=P-\tilde{P}, \pi=\tilde{P}-P_{h}$, $\alpha=\mathbf{u}-\tilde{\mathbf{u}}$ and $\sigma=\tilde{\mathbf{u}}-\mathbf{U}_{h}$. From theoretical results of mixed element (Ciarlet, 1978; Ewing, \& Wheeler, 1981; Wheeler, 1973), we get

$$
\begin{aligned}
& \|\alpha\|_{H(\operatorname{div} ; \Omega)}+\|\eta\|_{0} \leq K\|p\|_{k+3} h_{p}^{k+1}, \\
& \left\|\frac{\partial \alpha}{\partial t}\right\|_{H(\operatorname{div} ; \Omega)}+\left\|\frac{\partial \eta}{\partial t}\right\|_{0} \leq K\left\{\|p\|_{k+3}+\left\|\frac{\partial p}{\partial t}\right\|_{k+3}\right\} h_{p}^{k+1} .
\end{aligned}
$$

Subtracting (30a) $\left(t=t^{n+1}\right)$ from (32a), defining test function by $d_{t} \pi^{n}=\left\{\pi^{n+1}-\pi^{n}\right\} / \Delta t$, we have

$$
\begin{aligned}
& \left(d\left(C_{h}^{n}\right) d_{t} \pi^{n}, d_{t} \pi^{n}\right)+\left(\nabla \cdot \sigma^{n+1}, d_{t} \pi^{n}\right) \\
& =\left(\left(d\left(C_{h}^{n}\right)-d\left(c^{n+1}\right)\right) \frac{\partial \tilde{p}^{n+1}}{\partial t}, d_{t} \pi^{n}\right)-\left(d\left(C_{h}^{n}\right)\left[\frac{\tilde{p}^{n+1}-\tilde{p}^{n}}{\Delta t}-\frac{\partial \tilde{p}^{n+1}}{\partial t}\right], d_{t} \pi^{n}\right)-\left(d\left(c^{n+1}\right)\left[\frac{\partial p^{n+1}}{\partial t}-\frac{\partial \tilde{p}^{n+1}}{\partial t}\right], d_{t} \pi^{n}\right) .
\end{aligned}
$$

Combining (32b) $\left(t=t^{n+1}\right)$ and (30b), and taking $z_{h}=\sigma^{n+1}$, we have

$$
\left(d_{t}\left(a^{-1} \sigma^{n}\right), \sigma^{n+1}\right)-\left(\nabla \cdot \sigma^{n+1}, d_{t} \pi^{n}\right)=0 .
$$


Noting that

$$
d_{t}\left(a^{-1} \sigma^{n}, \sigma^{n}\right)=2\left(d_{t}\left(a^{-1} \sigma^{n}\right), \sigma^{n+1}\right)-\frac{1}{\Delta t}\left(a^{-1}\left(\sigma^{n+1}-\sigma^{n}\right),\left(\sigma^{n+1}-\sigma^{n}\right)\right),
$$

and $\frac{1}{\Delta t}\left(a^{-1}\left(\sigma^{n+1}-\sigma^{n}\right),\left(\sigma^{n+1}-\sigma^{n}\right)\right) \geq 0$, we have

$$
\frac{1}{2} d_{t}\left(a^{-1} \sigma^{n}, \sigma^{n}\right)-\left(\nabla \cdot \sigma^{n+1}, d_{t} \pi^{n}\right) \leq 0
$$

Adding (34) and (36),

$$
\begin{aligned}
& \left(d\left(C_{h}^{n}\right) d_{t} \pi^{n}, d_{t} \pi^{n}\right)+\frac{1}{2} d_{t}\left(a^{-1} \sigma^{n}, \sigma^{n}\right) \\
& \leq\left(\left(d\left(C_{h}^{n}\right)-d\left(c^{n+1}\right)\right) \frac{\partial \tilde{p}^{n+1}}{\partial t}, d_{t} \pi^{n}\right)-\left(d\left(C_{h}^{n}\right)\left[\frac{\tilde{p}^{n+1}-\tilde{p}^{n}}{\Delta t}-\frac{\partial \tilde{p}^{n+1}}{\partial t}\right], d_{t} \pi^{n}\right) \\
& \quad-\left(d\left(c^{n+1}\right)\left[\frac{\partial p^{n+1}}{\partial t}-\frac{\partial \tilde{p}^{n+1}}{\partial t}\right], d_{t} \pi^{n}\right) .
\end{aligned}
$$

Now each term on the right-hand side of (37) is estimated.

$$
\begin{aligned}
& \left|\left(\left(d\left(C_{h}^{n}\right)-d\left(c^{n+1}\right)\right) \frac{\partial \tilde{p}^{n+1}}{\partial t}, d_{t} \pi^{n}\right)\right| \leq \varepsilon\left\|d_{t} \pi^{n}\right\|^{2}+K\left\{\left\|\xi^{n}\right\|^{2}+h_{c}^{2(l+1)}+(\Delta t)^{2}\right\}, \\
& \left|\left(d\left(C_{h}^{n}\right)\left[\frac{\tilde{p}^{n+1}-\tilde{p}^{n}}{\Delta t}-\frac{\partial \tilde{p}^{n+1}}{\partial t}\right], d_{t} \pi^{n}\right)\right| \leq \varepsilon\left\|d_{t} \pi^{n}\right\|^{2}+K(\Delta t)^{2}, \\
& \left|\left(d\left(c^{n+1}\right)\left[\frac{\partial p^{n+1}}{\partial t}-\frac{\partial \tilde{p}^{n+1}}{\partial t}\right], d_{t} \pi^{n}\right)\right| \leq \varepsilon\left\|d_{t} \pi^{n}\right\|^{2}+K h_{p}^{2(k+1)} .
\end{aligned}
$$

Applying (38a)-(38c) and the positive definite condition (C) in (37), we have

$$
\left\|d_{t} \pi^{n}\right\|^{2}+d_{t}\left(a^{-1} \sigma^{n}, \sigma^{n}\right) \leq K\left\{\left\|\xi^{n}\right\|^{2}+h_{c}^{2(l+1)}+h_{p}^{2(k+1)}+(\Delta t)^{2}\right\} .
$$

Multiplying both sides of (39) by $\Delta t$, and summing them on $t(1 \leq n \leq L-1)$, we obtain

$$
\sum_{n=0}^{L-1}\left\|d_{t} \pi^{n}\right\|^{2} \Delta t+\left(a^{-1} \sigma^{L}, \sigma^{L}\right) \leq K\left\{\sum_{n=0}^{L}\left\|\xi^{n}\right\|^{2} \Delta t+h_{c}^{2(l+1)}+h_{p}^{2(k+1)}+(\Delta t)^{2}\right\} .
$$

The saturation equation is considered later. Subtracting (31) from (15) $\left(t=t^{n+1}\right)$ and using (19) $\left(t=t^{n+1}\right)$, we have

$$
\begin{aligned}
& \left(\phi \frac{\partial c^{n+1}}{\partial t}+\mathbf{u}^{n+1} \cdot \nabla c^{n+1}, v_{h}\right)-\left(\phi \frac{C_{h}^{n+1}-\hat{C}_{h}^{n}}{\Delta t}, v_{h}\right)+\left(D \nabla c^{n+1}, \nabla v_{h}\right)-\left(D \nabla C_{h}^{n+1}, \nabla v_{h}\right) \\
& +\left(b\left(c^{n+1}\right) \frac{\partial p^{n+1}}{\partial t}, v_{h}\right)-\left(b\left(C_{h}^{n}\right) \frac{P_{h}^{n+1}-P_{h}^{n}}{\Delta t}, v_{h}\right)+\left(D \frac{\partial c^{n+1}}{\partial \gamma},\left[v_{h}\right]\right)_{\Gamma}-\left(D B\left(C_{h}^{n}\right),\left[v_{h}\right]\right)_{\Gamma} \\
& =\left(g\left(c^{n+1}\right)-g\left(C_{h}^{n}\right), v_{h}\right) .
\end{aligned}
$$

Substituting the following results in (41),

$$
\begin{aligned}
& \left(\phi \frac{\partial c^{n+1}}{\partial t}+\mathbf{u}^{n+1} \cdot \nabla c^{n+1}, v_{h}\right)-\left(\phi \frac{C_{h}^{n+1}-\hat{C}_{h}^{n}}{\Delta t}, v_{h}\right) \\
& =\left(\left[\phi \frac{\partial c^{n+1}}{\partial t}+\mathbf{u}^{n+1} \cdot \nabla c^{n+1}-\phi \frac{c^{n+1}-\hat{c}^{n}}{\Delta t}\right], v_{h}\right)+\left(\phi\left[\frac{c^{n+1}-\hat{c}^{n}}{\Delta t}-\frac{C_{h}^{n+1}-\hat{C}_{h}^{n}}{\Delta t}\right], v_{h}\right) \\
& \quad+\left(\left(\mathbf{u}^{n+1}-\mathbf{U}_{h}^{n}\right) \cdot \nabla c^{n+1}, v_{h}\right), \\
& \left(D \frac{\partial c^{n+1}}{\partial \gamma},\left[v_{h}\right]\right)_{\Gamma}-\left(D B\left(C_{h}^{n}\right),\left[v_{h}\right]\right)_{\Gamma}=\left(D\left[\frac{\partial c^{n+1}}{\partial \gamma}-\frac{\partial c^{n}}{\partial \gamma}\right],\left[v_{h}\right]\right)_{\Gamma}+\left(D\left[\frac{\partial c^{n}}{\partial \gamma}-B\left(C_{h}^{n}\right)\right],\left[v_{h}\right]\right)_{\Gamma} \\
& +\left(D B\left(\zeta^{n}\right),\left[v_{h}\right]\right)_{\Gamma}+\left(D B\left(\xi^{n}\right),\left[v_{h}\right]\right)_{\Gamma},
\end{aligned}
$$


and taking $v_{h}=\xi^{n+1}$, using the elliptic projection (19), we have

$$
\begin{aligned}
& \left(\phi \frac{\xi^{n+1}-\xi^{n}}{\Delta t}, \xi^{n+1}\right)+\left(D \nabla \xi^{n+1}, \nabla \xi^{n+1}\right)+\lambda\left(\xi^{n+1}, \xi^{n+1}\right)+\left(D B\left(\xi^{n+1}\right),\left[\xi^{n+1}\right]\right)_{\Gamma} \\
& =\left(\left[\phi \frac{\partial c^{n+1}}{\partial t}+\mathbf{u}_{h}^{n+1} \cdot \nabla c^{n+1}\right]-\phi \frac{c^{n+1}-\hat{c}^{n}}{\Delta t}, \xi^{n+1}\right)+\left(\phi \frac{\hat{\xi}^{n}-\xi^{n}}{\Delta t}, \xi^{n+1}\right)-\left(\phi \frac{\xi^{n+1}-\hat{\zeta}^{n}}{\Delta t}, \xi^{n+1}\right) \\
& -\left(\left(\mathbf{u}^{n+1}-\mathbf{U}_{h}^{n}\right) \cdot \nabla c^{n+1}, \xi^{n+1}\right)+\left(g\left(c^{n+1}\right)-g\left(C_{h}^{n}\right), \xi^{n+1}\right)-\left(D\left[\frac{\partial c^{n+1}}{\partial \gamma}-\frac{\partial c^{n}}{\partial \gamma}\right],\left[\xi^{n+1}\right]\right)_{\Gamma} \\
& +\left(D\left[\frac{\partial c^{n}}{\partial \gamma}-B\left(C_{h}^{n}\right)\right],\left[\xi^{n+1}\right]\right)_{\Gamma}-\left(D B\left(\zeta^{n}\right),\left[\xi^{n+1}\right]\right)_{\Gamma}+\left(D B\left(\xi^{n+1}-\xi^{n}\right),\left[\xi^{n+1}\right]\right)_{\Gamma} \\
& -\left(b\left(c^{n+1}\right) \frac{\partial p^{n+1}}{\partial t}-b\left(C_{h}^{n}\right) \frac{P_{h}^{n+1}-P_{h}^{n}}{\Delta t}, \xi^{n+1}\right)+\lambda\left(\xi^{n+1}, \xi^{n+1}\right)+\lambda\left(\zeta^{n+1}, \xi^{n+1}\right) .
\end{aligned}
$$

The terms on the left-hand side of (43) are discussed as follows

$$
\begin{aligned}
& \left(\phi \frac{\xi^{n+1}-\xi^{n}}{\Delta t}, \xi^{n+1}\right)=\frac{1}{2 \Delta t}\left\{\left\|\phi^{1 / 2} \xi^{n+1}\right\|^{2}-\left\|\phi^{1 / 2} \xi^{n}\right\|^{2}\right\}+\frac{1}{2 \Delta t}\left\|\phi^{1 / 2}\left(\xi^{n+1}-\xi^{n}\right)\right\|^{2} \\
& \left(D \nabla \xi^{n+1}, \nabla \xi^{n+1}\right)+\lambda\left(\xi^{n+1}, \xi^{n+1}\right)+\left(D B\left(\xi^{n+1}\right),\left[\xi^{n+1}\right]\right)_{\Gamma} \geq M_{0}^{-1}\left\|\xi^{n+1}\right\|^{2},
\end{aligned}
$$

where $M_{0}$ is a positive constant.

Then the right-hand terms of (43) are discussed. Using (14), we have

$$
\begin{aligned}
& \left(D B\left(\xi^{n+1}-\xi^{n}\right),\left[\xi^{n+1}\right]\right)_{\Gamma} \leq M_{1}\left\|B\left(\xi^{n+1}-\xi^{n}\right)\right\|_{L^{2}(\Gamma)} \cdot\left\|\left[\xi^{n+1}\right]\right\|_{L^{2}(\Gamma)} \\
& \leq M_{1} H^{-3 / 2}\left\|\xi^{n+1}-\xi^{n}\right\| \cdot H^{1 / 2}\left\|\xi^{n+1}\right\| \mid \leq M_{1} H^{-2}\left\|\xi^{n+1}-\xi^{n}\right\|^{2}+\varepsilon\left\|\xi^{n+1}\right\| \|^{2}, \\
& \left(D\left[\frac{\partial c^{n+1}}{\partial \gamma}-\frac{\partial c^{n}}{\partial \gamma}\right],\left[\xi^{n+1}\right]\right)_{\Gamma} \leq M_{2}\left\|\frac{\partial c^{n+1}}{\partial \gamma}-\frac{\partial c^{n}}{\partial \gamma}\right\|_{L^{2}(\Gamma)} \cdot\left\|\left[\xi^{n+1}\right]\right\|_{L^{2}(\Gamma)} \\
& \leq M_{2} \Delta t H^{1 / 2} \varepsilon\left\|\xi^{n+1}\right\| \mid \leq M_{2}(\Delta t)^{2} H+\varepsilon\left\|\xi^{n+1}\right\| \|^{2}, \\
& \left(D\left[\frac{\partial c^{n}}{\partial \gamma}-B\left(C_{h}^{n}\right)\right],\left[\xi^{n+1}\right]\right)_{\Gamma} \leq M_{3} H^{2 m-1}+\left.\varepsilon\left\|\xi^{n+1}\right\|\right|^{2}, \\
& \left(D B\left(\zeta^{n}\right),\left[\xi^{n+1}\right]\right)_{\Gamma} \leq M_{4} H^{-2}\left\|\zeta^{n}\right\|^{2}+\varepsilon\left\|\xi^{n+1}\right\| \leq M_{4} H^{-2} h_{c}^{2(l+1)}+\varepsilon\left\|\xi^{n+1}\right\|^{2},
\end{aligned}
$$

where $M_{i}(i=1,2,3,4)$ are positive constants.

If the following constraint condition holds for sufficiently small $\Delta t$

$$
\Delta t \leq M_{1}^{-1} H^{2}, \quad h_{c}^{l+1}=o(H),
$$

then

$$
\frac{1}{2 \Delta t}\left\|\phi^{1 / 2}\left(\xi^{n+1}-\xi^{n}\right)\right\|^{2} \geq M_{1} H^{-2}\left\|\xi^{n+1}-\xi^{n}\right\|^{2}
$$

An induction hypothesis is introduced

$$
\sup _{0 \leq n \leq L}\left\|\sigma^{n}\right\|_{0, \infty} \rightarrow 0, \sup _{0 \leq n \leq L}\left\|\xi^{n}\right\|_{0, \infty} \rightarrow 0,\left(h_{p}, h_{c}\right) \rightarrow 0 .
$$


By using (48) we get estimates of the other terms on the right-hand side of (43) as follows

$$
\begin{aligned}
& \left|\left(\left[\phi \frac{\partial c^{n+1}}{\partial t}+\mathbf{u}_{h}^{n+1} \cdot \nabla c^{n+1}\right]-\phi \frac{c^{n+1}-\hat{c}^{n}}{\Delta t}, \xi^{n+1}\right)\right| \leq K\left\{\Delta t\left\|\frac{\partial^{2} c}{\partial \tau^{2}}\right\|_{L^{2}\left(t^{n}, t^{n+1} ; L^{2}(\Omega)\right)}^{2}+\left\|\xi^{n+1}\right\|^{2}\right\} \\
& \left|\left(\phi \frac{\hat{\xi}^{n}-\xi^{n}}{\Delta t}, \xi^{n+1}\right)\right| \leq K\left\|\xi^{n}\right\|^{2}+\varepsilon\left\|\nabla \xi^{n+1}\right\|^{2}, \\
& \left|\left(\phi \frac{\zeta^{n+1}-\hat{\zeta}^{n}}{\Delta t}, \xi^{n+1}\right)\right| \leq K\left\{(\Delta t)^{-1}\left\|\frac{\partial^{2} \zeta}{\partial t^{2}}\right\|_{L^{2}\left(t^{n}, t^{n+1} ; L^{2}(\Omega)\right)}^{2}+\left\|\xi^{n+1}\right\|^{2}+\left\|\xi^{n}\right\|^{2}\right\}+\varepsilon\left\|\nabla \xi^{n+1}\right\|^{2} \\
& \left.\left.\left|\left(b\left(c^{n+1}\right) \frac{\partial p^{n+1}}{\partial t}-b\left(C_{h}^{n}\right) \frac{P_{h}^{n+1}-P_{h}^{n}}{\Delta t}, \xi^{n+1}\right)\right| \quad||^{n}\right|^{n} \|^{2}+(\Delta t)^{2}+h_{c}^{2(l+1)}+h_{p}^{2(k+1)}\right\} \\
& \leq \varepsilon\left\|d_{t} \pi^{n}\right\|^{2}+K\left\{\left\|\xi^{n+1}\right\|^{2}+\|\left.\xi^{n}\right|^{2}\right. \\
& \left|\lambda\left(\xi^{n+1}, \xi^{n+1}\right)+\lambda\left(\zeta^{n+1}, \xi^{n+1}\right)\right| \leq K\left\{h_{c}^{2(l+1)}+\left\|\xi^{n+1}\right\|^{2}\right\} \\
& \left|\left(\left(\mathbf{u}^{n+1}-\mathbf{U}_{h}^{n}\right) \cdot \nabla c^{n+1}, \xi^{n+1}\right)\right| \leq K\left\{\left\|\sigma^{n+1}\right\|^{2}+h_{p}^{2(k+1)}+\left\|\xi^{n+1}\right\|^{2}\right\} \\
& \left|\left(g\left(c^{n+1}\right)-g\left(C_{h}^{n}\right), \xi^{n+1}\right)\right| \leq K\left\{(\Delta t)^{2}+h_{c}^{2(l+1)}+\left\|\xi^{n}\right\|^{2}+\left\|\xi^{n+1}\right\|^{2}\right\}
\end{aligned}
$$

Collecting all the estimates (44)-(49) for (43), we have

$$
\begin{aligned}
& \frac{1}{2 \Delta t}\left\{\left\|\phi^{1 / 2} \xi^{n+1}\right\|^{2}-\left\|\phi^{1 / 2} \xi^{n}\right\|^{2}\right\}+\frac{1}{M_{0}}\left\|\xi^{n+1}\right\|^{2} \\
& \leq \varepsilon\left\|\nabla \xi^{n+1}\right\|^{2}+\varepsilon\left\|d_{t} \pi^{n}\right\|^{2}+K\left\{(\Delta t)^{-1}\left\|\frac{\partial^{2} \zeta}{\partial t^{2}}\right\|_{L^{2}\left(t^{n}, t^{n+1} ; L^{2}(\Omega)\right)}^{2}+\Delta t\left\|\frac{\partial^{2} c}{\partial \tau^{2}}\right\|_{L^{2}\left(t^{n}, t^{n+1} ; L^{2}(\Omega)\right)}^{2}\right. \\
& \left.+h_{p}^{2(k+1)}+h_{c}^{2(l+1)}+(\Delta t)^{2}+(\Delta t)^{2} H+H^{-2} h_{c}^{2(l+1)}+H^{2 m+1}+\left\|\xi^{n+1}\right\|^{2}+\left\|\xi^{n}\right\|^{2}+\left\|\sigma^{n+1}\right\|^{2}\right\} .
\end{aligned}
$$

Applying (22) in (50), multiplying the resulting expression by $2 \Delta t$, summing them on $n(0 \leq n \leq L-1)$, and noting that $\xi^{0}=0$, we have

$$
\begin{aligned}
& \left\|\xi^{L}\right\|^{2}+\sum_{n=0}^{L-1}\left\|\xi^{n+1}\right\|^{2} \Delta t \\
& \leq \varepsilon \sum_{n=0}^{L-1}\left\|d_{t} \pi^{n}\right\|^{2} \Delta t+K\left\{\sum_{n=0}^{L}\left\{\left\|\xi^{n}\right\|^{2}+\left\|\sigma^{n}\right\|^{2}\right\} \Delta t+(\Delta t)^{2}+h_{p}^{2(k+1)}+h_{c}^{2(l+1)}+H^{-2} h_{c}^{2(l+1)}+H^{2 m+1}\right\} .
\end{aligned}
$$

Then it follows from (40) and (51),

$$
\begin{aligned}
& \sum_{n=0}^{L-1}\left\|d_{t} \pi^{n}\right\|^{2} \Delta t+\sum_{n=0}^{L}\left\|\xi^{n}\right\|^{2} \Delta t+\left\|\sigma^{L}\right\|^{2}+\left\|\xi^{L}\right\|^{2} \\
& \leq K\left\{\sum_{n=0}^{L}\left\{\left\|\xi^{n}\right\|^{2}+\left\|\sigma^{n}\right\|^{2}\right\} \Delta t+(\Delta t)^{2}+h_{p}^{2(k+1)}+h_{c}^{2(l+1)}+H^{-2} h_{c}^{2(l+1)}+H^{2 m+1}\right\} .
\end{aligned}
$$

Using Gronwall Lemma, we have

$$
\begin{aligned}
& \sum_{n=0}^{L-1}\left\|d_{t} \pi^{n}\right\|^{2} \Delta t+\sum_{n=0}^{L}\left\|\xi^{n}\right\|^{2} \Delta t+\left\|\sigma^{L}\right\|^{2}+\left\|\xi^{L}\right\|^{2} \\
\leq & K\left\{(\Delta t)^{2}+h_{p}^{2(k+1)}+h_{c}^{2(l+1)}+H^{-2} h_{c}^{2(l+1)}+H^{2 m+1}\right\} .
\end{aligned}
$$

It remains to testify the hypothesis (48). As $n=0$, it is true because of $\xi^{0}=0$ and $\sigma^{0}=0$. If (48) holds for $0 \leq n \leq L-1$. For $n=L$ and $k, l \geq 1$, we get the conclusion from (53) and the following constraint

$$
\Delta t=o\left(h_{c}^{3 / 2}\right), h_{c} \sim h_{p}, H^{-1} h_{c}^{l+1}=o\left(h_{c}^{3 / 2}\right), H^{m+1 / 2}=o\left(h_{c}^{3 / 2}\right) .
$$


From (53), (22) and (33), we obtain the following convergence theorem.

Theorem 1 Suppose that (27) and (28) are positive definite (C), and suppose that exact solutions are suitably regular, $p \in L^{\infty}\left(J ; W^{k+3}(\Omega)\right), c \in L^{\infty}\left(J ; W^{l+1}(\Omega)\right), \frac{\partial^{2} c}{\partial \tau^{2}} \in L^{\infty}\left(J ; L^{\infty}(\Omega)\right)$. Numerical solutions are obtained in parallel by using the procedures (30) and (31) on $\Omega_{1}, \Omega_{2}$. If the constraints (46) and (54) hold, then we have the following estimates for $k, l \geq 1$,

$$
\begin{aligned}
& \left\|p-P_{h}\right\|_{L^{\infty}(J ; W)}+\left\|d_{t}\left(p-P_{h}\right)\right\|_{L^{\infty}\left(J ; L^{2}(\Omega)\right)}+\left\|\mathbf{u}-\mathbf{U}_{h}\right\|_{\bar{L}^{\infty}(J ; V)}+\left\|c-C_{h}\right\|_{L_{L^{\infty}\left(J ; L^{2}(\Omega)\right)}} \\
& +\left\|c-C_{h}\right\|_{\bar{L}^{2}(J ; \bar{W})} \\
& \leq K^{*}\left\{\Delta t+H^{m+1 / 2}+h_{p}^{k+1}+h_{c}^{l+1}+H^{-1} h_{c}^{l+1}\right\}
\end{aligned}
$$

where $\|g\|_{\bar{L}^{\infty}(J ; X)}=\sup _{n \Delta t \leq T}\left\|g^{n}\right\|_{X},\|g\|_{\bar{L}^{2}\left(J ; L^{2}(\Omega)\right)}=\sup _{N \Delta t \leq T}\left\{\sum_{n=0}^{N}\left\|g^{n}\right\|^{2} \Delta t\right\}^{1 / 2},\|g\|_{\bar{L}^{2}(J ; \bar{W})}=\sup _{N \Delta t \leq T}\left\{\sum_{n=0}^{N}\left\|g^{n}\right\| \|^{2} \Delta t\right\}^{1 / 2}$, the constant $K^{*}$ depends on $p, c$ and their derivatives. Note. The method discussed above can be used by decomposing the whole domain $\Omega$ into several subdomains such as four subdomains in Fig. $2, \Omega=\bigcup_{i=1}^{4} \Omega_{i}$. Therefore, it is important in solving actual problems numerically.

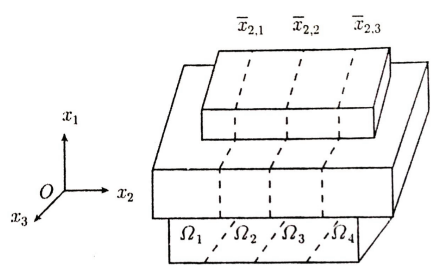

Figure 2. Subdomains decomposition of a 3-D domain, $\Omega=\bigcup_{i=1}^{4} \Omega_{i}$

\section{Numerical Experiment}

In this section we give a numerical example to testify the parallel scheme. Consider the following model

$$
\begin{gathered}
\frac{\partial p}{\partial t}+\frac{\partial}{\partial x}\left((1+p) \frac{\partial p}{\partial x}\right)=g(x, t), 0<x<1,0<t \leq T, \\
\mathbf{u}=-(1+p) \frac{\partial p}{\partial x}, 0<x<1,0<t \leq T, \\
(1+p) \frac{\partial c}{\partial t}+\mathbf{u} \frac{\partial c}{\partial x}-\frac{\partial}{\partial x}\left(D(x, t) \frac{\partial c}{\partial x}\right)=f(c, x, t), 0<x<1,0<t \leq T, \\
p(x, 0)=x^{2}, 0 \leq x \leq 1, \\
c(x, 0)=\cos (2 \pi), 0 \leq x \leq 1, \\
\frac{\partial p}{\partial x}(0, t)=0, \frac{\partial p}{\partial x}(1, t)=2 e^{t}, 0 \leq t \leq T, \\
\frac{\partial c}{\partial x}(0, t)=\frac{\partial c}{\partial x}(1, t)=0,0 \leq t \leq T .
\end{gathered}
$$

Take $D(x, t)=0.01 x^{2} e^{2 t}, c=e^{t} \cos (2 \pi x), p=x^{2} e^{t}, f=\left(1+x^{2} e^{t}\right) e^{t} \cos (2 \pi x)+4 \pi x e^{2 t}\left(1+x^{2} e^{t}\right) \sin (2 \pi x)+0.04 \pi x e^{3 t} \sin (2 \pi x)+$ $0.04 \pi^{2} x^{2} e^{3 t} \cos (2 \pi x), H=4 h, \Delta t_{c}=\frac{1}{12} h^{2}, \Delta t_{p}=4 \Delta t_{c}, T=0.25$. Consider a simple test space with $m=2, k=1$ and $l=1$. The spacial interval $[0,1]$ is divided into two subdomians $\left[0, \frac{1}{2}\right]$ and $\left[\frac{1}{2}, 1\right]$. Absolute errors of the saturation $c$ and the pressure $p$ at different nodes are shown in Table 1 and Table 2 .

Table 1. Error of $c,\left\|c-C_{h}\right\|$

\begin{tabular}{ccccccc}
\hline & $x=0.05$ & $x=0.25$ & $x=0.45$ & $x=0.55$ & $x=0.75$ & $x=0.95$ \\
\hline$h=1 / 40$ & $64.3205 e-3$ & $1.1178 e-3$ & $64.1035 e-3$ & $64.3205 e-3$ & $1.1178 e-3$ & $64.3205 e-3$ \\
\hline$h=1 / 80$ & $14.9841 e-3$ & $0.0773 e-3$ & $14.6352 e-3$ & $14.9841 e-3$ & $0.0773 e-4$ & $14.6351 e-3$ \\
\hline$h=1 / 160$ & $3.6547 e-3$ & $0.0048 e-3$ & $3.6588 e-3$ & $3.6547 e-3$ & $0.0051 e-3$ & $3.6588 e-3$ \\
\hline
\end{tabular}


Table 2. Error of $p,\left\|p-P_{h}\right\|$

\begin{tabular}{ccccccc}
\hline & $x=0.05$ & $x=0.25$ & $x=0.45$ & $x=0.55$ & $x=0.75$ & $x=0.95$ \\
\hline$h=1 / 40$ & $51.0082 e-3$ & $11.2156 e-3$ & $51.3174 e-3$ & $51.0882 e-3$ & $11.2156 e-3$ & $51.3174 e-3$ \\
\hline$h=1 / 80$ & $12.1448 e-3$ & $0.8011 e-3$ & $12.2184 e-3$ & $12.1448 e-3$ & $0.8010 e-4$ & $12.2184 e-3$ \\
\hline$h=1 / 160$ & $2.9622 e-3$ & $0.0499 e-3$ & $2.9801 e-3$ & $2.9622 e-3$ & $0.0499 e-3$ & $2.9801 e-3$ \\
\hline
\end{tabular}

From Table 1 and Table 2 we conclude that numerical data are consistent with theoretical result, i.e., numerical errors are measured about by $O\left(\Delta t+h^{2}\right)$.

Numerical approximations of normal derivative at interior boundary $\frac{\partial c}{\partial x}(0.5)=e^{T} \sin (\pi)=0$ is shown in Table 3 . The approximation at interior boundary is quite well so that the parallel computations on subdomains have high order accuracy.

Table 3. Error estimates of $B$

\begin{tabular}{cc}
\hline \multicolumn{1}{c}{$B$} \\
\hline$h=1 / 40$ & $3.1746 e-15$ \\
\hline$h=1 / 80$ & $2.8594 e-14$ \\
\hline$h=1 / 160$ & $4.1065 e-13$ \\
\hline
\end{tabular}

Domain decomposition with two subdomains and whole domain scheme have similar discretizations and their computational efficiency are compared. Computational time cost of different schemes under the fixed error level are shown in Table 4 (unit: second). We can see that the computational scale becomes large and domain decomposition is more efficient as $h \rightarrow 0$. One reason is that domain decomposition can solve the problem in parallel and saves computational time. The other is the computational scale of each subdomain becomes a half of the computation of whole domain. It is hoped that domain decomposition is used in actual applications efficiently and can solve the complicated problem quickly as the partition becomes refined and the number of subdomains becomes large.

Table 4. Time cost comparison (unit: second)

\begin{tabular}{lcc}
\hline & Domain decomposition & Whole domain computation \\
\hline$h=1 / 40$ & 0.8532 & 1.7364 \\
\hline$h=1 / 80$ & 1.8976 & 4.2377 \\
\hline$h=1 / 160$ & 7.4303 & 31.2657 \\
\hline$h=1 / 320$ & 166.9077 & 652.3577 \\
\hline
\end{tabular}

\section{The Parallel Algorithm and Analysis for Slightly Compressible SeepageDisplacement with Multicomponents}

In numerical simulation of enhanced oil recovery (chemical recovery), we should consider multicomponent seepage displacement and formulate its mathematical model by the following nonlinear system of partial differential equations with initial-boundary value conditions (Douglas, \& Roberts, 1983; Yuan, 2013)

$$
\begin{gathered}
d(c) \frac{\partial p}{\partial t}+\nabla \cdot \mathbf{u}=q(X, t), X=\left(x_{1}, x_{2}, x_{3}\right)^{T} \in \Omega, t \in J=(0, T], \\
\mathbf{u}=a(c) \nabla p, X \in \Omega, t \in J \\
\phi(X) \frac{\partial c_{\alpha}}{\partial t}+b_{\alpha}(c) \frac{\partial p}{\partial t}+\mathbf{u} \cdot \nabla c_{\alpha}-\nabla \cdot\left(D \nabla c_{\alpha}\right)=g\left(X, t, c_{\alpha}\right), X \in \Omega, t \in J, \alpha=1,2, \cdots, n_{c} .
\end{gathered}
$$

The pressure and the saturation of $\alpha$-th component are denoted by $p(X, t)$ and $c_{\alpha}(X, t), \alpha=1,2, \cdots, n_{c} . n_{c}$ is the number of components. Since $\sum_{\alpha=1}^{n_{c}} c_{\alpha}(X, t)=1$, so only $n_{c}-1$ component saturations are independent. In this section we aim to find numerical solutions of the pressure $p(X, t)$ and the saturations $c(X, t)=c_{\alpha}(X, t), \alpha=1,2, \cdots, n_{c}$.

The parallel algorithm of characteristic mixed element is defined as follows. If numerical solutions at $t^{n},\left\{P_{h}^{n}, \mathbf{U}_{h}^{n}, C_{\alpha, h}^{n}, \alpha=\right.$ $\left.1,2, \cdots, n_{c}-1\right\} \in W_{h} \times V_{h} \times N_{h}^{n_{c}-1}$, are given, then $\left\{P_{h}^{n+1}, \mathbf{U}_{h}^{n+1}\right\} \in W_{h} \times V_{h}, C_{\alpha, h}^{n+1}\left(\alpha=1,2, \cdots, n_{c}-1\right) \in N_{h}^{n_{c}-1}$ are computed 
by

$$
\begin{aligned}
& \mathbf{U}_{h}^{0}=\tilde{\mathbf{u}}^{0}, P_{h}^{0}=\tilde{p}^{0}, \\
& \left(d\left(C_{h}^{n}\right) \frac{P_{h}^{n+1}-P_{h}^{n}}{\Delta t}, w_{h}\right)+\left(\nabla \cdot \mathbf{U}_{h}^{n+1}, w_{h}\right)=\left(q, w_{h}\right), \forall w_{h} \in W_{h}, \\
& \left(a\left(C_{h}^{n}\right) \mathbf{U}_{h}^{n+1}, z_{h}\right)-\left(\nabla \cdot z_{h}, P_{h}^{n+1}\right)+\sum_{i=1}^{2}\left(P_{h}^{n+1}, z_{h}^{(i)} \cdot n_{i}\right)_{\Gamma}=0, \forall z_{h} \in V_{h}, \\
& \left(\beta, \mathbf{U}_{1}^{n+1} \cdot n_{1}+\mathbf{U}_{2}^{n+1} \cdot n_{2}\right)_{\Gamma}=0, \forall \beta \in \Lambda_{h}, \\
& C_{\alpha, h}^{0}=\tilde{c}_{\alpha}^{0}, \\
& \left(\phi \frac{C_{\alpha, h}^{n+1}-\hat{C}_{\alpha, h}^{n}}{\Delta t}, v_{h}\right)+\left(D \nabla C_{\alpha, h}^{n+1}, \nabla v_{h}\right)+\left(b_{\alpha}\left(C_{\alpha, h}^{n}\right) \frac{P_{h}^{n+1}-P_{h}^{n}}{\Delta t}, v_{h}\right)+\left(D B\left(C_{\alpha, h}^{n}\right),\left[v_{h}\right]\right)_{\Gamma} \\
& =\left(g\left(C_{\alpha, h}^{n}\right), v_{h}\right), v_{h} \in N_{h}, \alpha=1,2, \cdots, n_{c} .
\end{aligned}
$$

After a similar analysis, we derive the following convergence statement.

Theorem 2. Suppose that (60) and (61) are positive definite and exact solutions are suitably smooth, $p \in L^{\infty}\left(J ; W^{k+3}(\Omega)\right), c_{\alpha} \in$ $L^{\infty}\left(J ; W^{l+1}(\Omega)\right), \frac{\partial^{2} c_{\alpha}}{\partial \tau_{\alpha}^{2}} \in L^{\infty}\left(J ; L^{\infty}(\Omega)\right), \alpha=1,2, \cdots, n_{c}$. Numerical solutions are computed by the parallel procedures (62) and (63) in parallel on $\Omega_{1}, \Omega_{2}$. If (46) and (54) hold, then we have the following error estimate for $k, l \geq 1$,

$$
\begin{aligned}
& \left\|p-P_{h}\right\|_{\bar{L}^{\infty}(J ; W)}+\left\|d_{t}\left(p-P_{h}\right)\right\|_{\bar{L}^{\infty}\left(J ; L^{2}(\Omega)\right)}+\left\|\mathbf{u}-\mathbf{U}_{h}\right\|_{\bar{L}^{\infty}(J ; V)}+\sum_{\alpha=1}^{n_{c}-1}\left\|c_{\alpha}-C_{\alpha, h}\right\|_{\bar{L}^{\infty}\left(J ; L^{2}(\Omega)\right)}+\sum_{\alpha=1}^{n_{c}-1}\left\|c_{\alpha}-C_{\alpha, h}\right\|_{\bar{L}^{2}(J ; \bar{W})} \\
& \leq K^{* *}\left\{\Delta t+H^{m+1 / 2}+h_{p}^{k+1}+h_{c}^{l+1}+H^{-1} h_{c}^{l+1}\right\},
\end{aligned}
$$

where the constant $K^{* *}$ depends on $p, c_{\alpha}\left(\alpha=1,2, \cdots, n_{c}-1\right)$ and their derivatives.

\section{Conclusions and Discussions}

A domain decomposition method of modified characteristic mixed finite element is discussed for slightly compressible oilwater seepage displacement in this paper. In $\S 1$ Induction, the mathematical model, physical background and some related research studies are stated. In $\$ 2$ some preliminary work and elementary error estimates are given. In $\S 3$ we formulate the parallel procedures of characteristic mixed finite element. Numerical analysis and optimal order $l^{2}$ norm estimate are shown in §4. Finally a numerical example is given to show the efficiency and application in $\S 5$. In $\S 6$ we construct the domain decomposition algorithm of characteristic mixed element for multi-component seepage displacement and give numerical analysis. In this paper some features are concluded as follows. (I) This method is suitable to solve oil reservoir numerical simulation on three-dimensional irregular geometric region especial enhanced oil numerical computation. (II) By using mixed finite element, the method improves an order accuracy to compute Darcy velocity. It is most important in numerical simulation of oil reservoir. (III) The method of characteristics can confirm strong stability of numerical simulation at the fronts, and can avoid numerical dispersion and nonphysical oscillation. It can adopt large-time step but can obtain small time truncation error and can improve the computation accuracy. The parallel scheme is quite efficient in computing the saturation because the whole computation on a large-scaled domain is decomposed into several smallscaled computations on subdomains. (IV) It is easy to compute numerical simulation in parallel with high order accuracy on modern parallel machine.

Acknowledgements The authors express their deep appreciation to Prof. J. Douglas Jr, and Prof. Jiang Lishang for their helpful suggestions in the serial research of numerical simulation of oil reservoir.

\section{References}

Brezzi, F. (1974). On the existence, uniqueness and approximation of saddle-point problems arising from lagrangian multipliers. RAIRO Anal. Numer., 2, 129-151.

Ciarlet, P. G. (1978). The finite element method for elliptic problems. Amsterdam: North-Holland.

Dawson, C. N., \& Du, Q. (1990). A finite element domain decomposition method for parabolic equations. Rice Technical'report TR90-21, Dept. of Mathematical Sciences, Rice University, Houston, Texas.

Dawson, C. N., Du, Q., \& Dupont, T. F. (1991). A finite difference domain decomposition algorithm for numerical solution of the heat equation. Math. Comp., 57(195), 63-71. 
Dawson, C. N., \& Dupont, T. F. (1992). Explicit/Implicit conservative Galerkin domain decomposition procedures for parabolic problems. Math. Comp., 58(197), 21-34.

Dawson, C. N., \& Dupont, T. F. (1994). Explicit/Implicit, conservative domain decomposition procedures for parabolic problems based on block-centered finite differences. SIAM J. Numer. Anal., 31(4), 1045-1061.

Douglas, Jr. J., \& Roberts, J. E. (1983). Numerical methods for a model for compressible miscible displacement in porous media. Math. Comp., 41(164), 441-459.

Douglas, Jr. J., \& Russell, T. F. (1982). Numerical method for convection-dominated diffusion problems based on combining the method of characteristics with finite element or finite difference procedures. SIAM J. Numer. Anal., 19(5), 871-885.

Douglas, Jr. J., \& Yuan, Y. R. (1986). Numerical simulation of immiscible flow in porous media based on combining the method of characteristics with mixed finite element procedure. The IMA Vol. in Math. and its Appl., 11, 119-131.

Ewing, R. E. (1983). The Mathematics of Reservoir Simulation. SIAM, Philadelphia.

Ewing, R. E., \& Russell, T. F. (1982). Efficient time-stepping methods for miscible displacement problems in porous media. SIAM J Numer. Anal., 19(1), 1-67.

Ewing, R. E., Russell, T. F., \& Wheeler, M. F. (1984). Convergence analysis of an approximation of miscible displacement in porous media by mixed finite elements and a modified method of characteristics. Comp. Meth. Appl. Mech. Eng., 47(1-2), 73-92.

Ewing, R. E., \& Wheeler, M. F. (1981). Galerkin methods for miscible displacement problems with point sources and sinks-unit mobility ratio case. Proc. Special Year in Numerical Anal., Lecture Notes \#20, Univ. Maryland, College Park, 151-174.

Ewing, R. E., Yuan, Y. R., \& Li, G. (1989). Time stepping along characteristics of a mixed finite element approximation for compressible flow of contamination by nuclear waste disposal in porous media. SIAM J. Numer. Anal., 26(6), 1513-1524.

Raviart, P. A., \& Thomas, J. M. (1977). A mixed finite element method for second order elliptic problems, in: Mathematical Aspects of the Finite Element Method. Lecture Notes in Mathematics, 606, Berlin: Springer-Verlag.

Russell, T. F. (1985). Time stepping along characteristics with incomplete interaction for a Galerkin approximation of miscible displacement in porous media. SLAM J. Numer. Anal., 22(5), 970-1013.

Shen, P. P., Liu, M. X., \& Tang, L. (2002). Mathematical model of petroleum exploration and development. Science Press, Beijing.

Wheeler, M. F. (1973). A prior $L^{2}$-error estimates for Galerkin approximations to parabolic differential equations. SIAM J. Numer. Anal., 10(4), 723-759.

Yuan, Y. R. (1992). Time stepping along characteristics for the finite element approximation of compressible miscible displacement in porous media. Math. Numer. Sinica, 14(4), 385-400.

Yuan, Y. R. (1993). Finite difference methods for a compressible miscible displacement problem in porous media. Math. Numer. Sinica, 15(1), 16-28.

Yuan, Y. R. (1999). The characteristic finite difference fractional steps method for compressible two-phase displacement problem. Sci. China Math., 42(1), 48-57.

Yuan, Y. R. (2001). Characteristic finite difference fractional steps method for three-dimensional compressible multicomponent displacement problem. Acta Math. Appl. Sin., 24(2), 242-249.

Yuan, Y. R. (2003). The modified method of characteristics with finite element operator-splitting procedures for compressible multi-component displacement problem. J Syst. Sci. Complex., 16(1), 30-45.

Yuan, Y. R. (2003). The upwind finite difference fractional steps methods for two-phase compressible flow in porous media. Numer. Meth. Part. D. E., 19(1), 67-88.

Yuan, Y. R. (2013). Theory and application of reservoir numerical simulation. Science Press, Beijing.

Yuan, Y. R., Chang, L., Li, C. F., \& Sun, T. J. (2015). Domain decomposition modified with characteristic finite element method for numerical simulation of semiconductor transient problem of heat conduction. J Mathematics Research, 7(3), 1-14. 
Yuan, Y. R., Chang, L., Li, C. F., \& Sun, T. J. (2015). Theory and applications of domain decomposition with characteristic mixed finite element of three-dimensional semiconductor transient problem of heat conduction. Far East J Appl. Math., 92(1), 51-80.

Yuan, Y. R., Chang, L., Li, C. F., \& Sun, T. J. (2015). The modified method of characteristics with mixed finite element domain decomposition procedures for the enhanced oil recovery simulation. Far East J Appl. Math., 93(2), 123-152. http://dx.doi.org/10.17654/FJAMNov2015-123-152

\section{Copyrights}

Copyright for this article is retained by the author(s), with first publication rights granted to the journal.

This is an open-access article distributed under the terms and conditions of the Creative Commons Attribution license (http://creativecommons.org/licenses/by/4.0/). 